\title{
The impact of production of extended- spectrum $\beta$-lactamases on the 28-day mortality rate of patients with Proteus mirabilis bacteremia in Korea
}

\author{
Jin Young Ahn ${ }^{1,2}$, Hea Won Ann ${ }^{2,3}$, Yongduk Jeon ${ }^{2,3}$, Mi Young Ahn ${ }^{2,3}$, Dong Hyun Oh ${ }^{2,3}$, Yong Chan Kim ${ }^{2,3}$, \\ Eun Jin Kim ${ }^{2,3}$, Je Eun Song ${ }^{4}$, In Young Jung ${ }^{2,3}$, Moo Hyun Kim² ${ }^{2}$, Wooyoung Jeong ${ }^{2}$, Nam Su Ku ${ }^{2,3,6 *}$, \\ Su Jin Jeong ${ }^{2,3}$, Jun Yong Choi ${ }^{2,3}$, Dongeun Yong ${ }^{5}$, Young Goo Song ${ }^{2,3}$ and June Myung Kim ${ }^{2,3}$
}

\begin{abstract}
Background: The incidence of Proteus mirabilis antimicrobial resistance, especially that mediated by extended-spectrum $\beta$-lactamases (ESBLs), has increased. We investigated the impact of ESBL production on the mortality of patients with P. mirabilis bacteremia in Korea.

Methods: Patients diagnosed with P. mirabilis bacteremia between November 2005 and December 2013 at a 2000-bed tertiary care center in South Korea were included in this study. Phenotypic and molecular analyses were performed to assess ESBL expression. Characteristics and treatment outcomes were investigated among ESBL-producing and non-ESBL-producing P. mirabilis bacteremia groups. A multivariate analysis of 28-day mortality rates was performed to evaluate the independent impact of ESBLs.

Results: Among 62 P. mirabilis isolates from 62 patients, 14 expressed ESBLs (CTX-M, 2; TEM, 5; both, 6 ; other, 1), and the 28-day mortality rate of the 62 patients was $17.74 \%$. No clinical factor was significantly associated with ESBL production. The 28-day mortality rate in the ESBL-producing group was significantly higher than that in the non-ESBL-producing group (50\% vs. $8.3 \%, p=0.001)$. A multivariate analysis showed that ESBL production (odds ratio [OR], 11.53, 95\% confidence interval [Cl], 2.11-63.05, $p=0.005$ ) was independently associated with the 28-day mortality rate in patients with P. mirabilis bacteremia.
\end{abstract}

Conclusions: ESBL production is significantly associated with mortality in patients with bacteremia caused by P. mirabilis. Rapid detection of ESBL expression and prompt appropriate antimicrobial therapy are required to reduce mortality caused by $P$. mirabilis bacteremia.

Keywords: Proteus Mirabilis, Bacteremia, Extended-spectrum $\beta$-lactamase, Mortality

\section{Background}

Proteus mirabilis is not a common cause of bloodstream infections in normal hosts, accounting for $1-3 \%$ of all episodes of bacteremia. [1-3] However, $P$. mirabilis is an important causative pathogen of various community-

\footnotetext{
* Correspondence: smileboy9@yuhs.ac

${ }^{2}$ Department of Internal Medicine, Yonsei University College of Medicine, Seoul, South Korea

${ }^{3}$ AIDS Research Institute, Yonsei University College of Medicine, Seoul, South Korea

Full list of author information is available at the end of the article
}

and healthcare-associated infections, such as wound infections, primary bacteremia, pneumonia and urinary tract infections, particularly among patients with anatomical or functional urinary tract abnormalities or indwelling urinary catheters [4-6].

The incidence of antimicrobial resistance of $P$. mirabilis has increased, and this can negatively affect prognosis. [7] The prevalence of multidrug-resistant (MDR) strains producing extended-spectrum $\beta$-lactamases (ESBLs), AmpC $\beta$-lactamases or carbapenemases has increased worldwide. [8-13] Among MDR isolates, ESBL-producing strains are 
the most frequent. $[5,9,14]$ Previous studies reported that infections caused by MDR P. mirabilis strains are associated with higher rates of antibiotic treatment failure and mortality. $[5,8,14]$ However, the treatment outcomes of MDR and non-MDR $P$. mirabilis infections are reported to be similar in several reports. $[9,15]$ The impact of MDR in P. mirabilis infections on treatment outcomes is thus unclear.

In Korea, the clinical aspects of $P$. mirabilis bacteremia have not been assessed, although microbiological data and molecular analyses of ESBLs from $P$. mirabilis strains have been reported. [16-18] Therefore, further study of the effects of MDR on clinical outcomes is needed. To this end, we investigated the clinical characteristics and antimicrobial susceptibility profile of $P$. mirabilis bacteremia isolates in Korea. We also evaluated the treatment outcomes of patients with ESBL-producing P. mirabilis bacteremia, in particular the impact of ESBL expression on the mortality rate of patients with $P$. mirabilis bacteremia.

\section{Methods}

\section{Study design and patients}

A retrospective cohort study was conducted at a 2000bed, tertiary-care medical center in Seoul, South Korea. Microbiology laboratory databases were searched to identify all blood cultures positive for $P$. mirabilis among hospitalized patients older than 18 years from November 2005 to December 2013. Among the identified patients, only those with stored $P$. mirabilis isolate samples were included in the study, and their medical records were reviewed. For patients with more than one episode of $P$. mirabilis bacteremia, only data relevant to the first episode were analyzed. Isolates were divided into two groups according to ESBL production (the ESBLproducing and non-ESBL-producing groups). Microbiological and clinical factors were compared between the two groups to evaluate the factors and outcomes associated with ESBL production in $P$. mirabilis bacteremia. The study was approved by the Institutional Review Board (IRB) of Yonsei University Health System Clinical Trial Center. Since the study was retrospective, and the data of the subjects were anonymized, the IRB waived the requirement for written informed consent from the patients.

\section{Microbiological tests and molecular detection of ESBL}

P. mirabilis was identified using either the ATB $32 \mathrm{GN}$ or VITEK 2 system (bioMérieux, Marcy-L'Étoile, France). Antimicrobial susceptibility was determined using the disk-diffusion method or VITEK-2 N131 card (bioMérieux, Hazelwood, MO, USA). The results were interpreted according to the Clinical and Laboratory Standards Institute (CLSI) 2014 guidelines. [19] P. mirabilis isolates were stored in skim milk at $-70{ }^{\circ} \mathrm{C}$ until further examination. In our institution, until 2010, ESBL production was assessed using the double-disk potentiation test. However, from 2011, ESBL production was no longer reported, in accordance with the revised CLSI 2011 guidelines. [20] Thus, we retrospectively performed the double-disk potentiation test to identify ESBL-production. Briefly, each isolate was subcultured twice from the skim milk prior to being tested. All 62 P. mirabilis isolates were inoculated onto the plates of Muller-Hilton agar (Becton Dickinson, Cockeysville, Md.) using ceftazidime and cefotaxime disks with and without clavulanic acid. The interpretation was followed by CLSI 2014 guidelines. [19] Genes encoding ESBLs $\left(b l a_{\mathrm{TEM}}, b l a_{\mathrm{SHV}}\right.$ and $\left.b l a_{\mathrm{CTX}-\mathrm{M}}\right)$ were detected by polymerase chain reaction amplification using previously reported primers and reaction conditions [21-23].

\section{Collected data and definitions}

The data collected included age, sex, date of culture, possible source of bacteremia, underlying diseases, various predisposing factors, laboratory data at the time of bacteremia diagnosis, severity of disease calculated by the sequential organ failure (SOFA) score and acute physiological and chronic health evaluation (APACHE) II score, treatment outcomes, antimicrobial therapy regimen and results of antimicrobial susceptibility testing.

Bacteremia was defined as the isolation of $P$. mirabilis from at least one separately obtained blood culture with clinical symptoms and signs compatible with infection. [24] Comorbidities were defined according to the International Classification of Disease, 10th Revision. [25] Predisposing conditions were taken into consideration only if they occurred within 1 month before the bacteremia. Previous exposure to specific antibiotics was considered in the analysis only if the antibiotics had been administered for at least 3 consecutive days within 1 month before the bacteremia.

After performing blood cultures, patients were promptly treated with empirical antibiotics within $24 \mathrm{~h}$ according to their predicted focus of infection until the susceptibility profiles of the isolates had been determined. The appropriateness of the prescribed antimicrobial therapy was evaluated retrospectively at the time of the bacteremia episode. Antimicrobial therapy was considered inappropriate if the isolated P. mirabilis did not show susceptibility to all administered antibiotics in vitro. Death within 28 days after bacteremia was regarded as being associated with $P$. mirabilis bacteremia unless definite clinical data suggested another cause of death.

\section{Statistical analysis}

All statistical analyses were performed using the SPSS software, version 21 (SPSS; IBM Corp., Armonk, NY, USA) Continuous variables are presented as means \pm 
[standard deviation (SD)] or medians [interquartile range (IQR)], and categorical variables are presented as numbers and percentages. To compare the two groups, the Student's $t$-test or Mann-Whitney $U$-test, depending on the validity of the normality assumption, was used for continuous variables. The chi-squared test or Fisher's exact test was used to assess categorical variables. Multivariate analysis was performed using logistic regression to identify factors that independently and significantly affected outcomes. Variables with a $p$ value $<0.05$ in the univariate analysis were considered for inclusion in a multivariate model, and the final variables for inclusion in the multivariate model were selected via the backward likelihood ratio test. Survival analysis was performed using the Kaplan-Meier method, and comparison between the two groups was performed by log-rank test. Values of $p<0.05$ were considered to indicate significance.

\section{Results}

Prevalence of ESBL production in P. mirabilis bacteremia From November 2005 to December 2013, a total of 85 patients with $P$. mirabilis bacteremia were identified: 5 in 2005/2006, 7 in 2007, 9 in 2008, 8 in 2009, 10 in 2010, 18 in 2011, 17 in 2012 and 11 patients in 2013. Among them, 62 patients with stored bacterial strain samples were included in the analysis. Fourteen of 62 patients harbored ESBL-producing $P$. mirabilis strains (prevalence, 22.6\%).

\section{Antimicrobial susceptibility of $P$. mirabilis}

The in vitro antimicrobial susceptibilities of the $P$. mirabilis isolates are shown in Fig. 1. Most isolates were susceptible to meropenem (93.5\%), piperacillin/tazobactam (98.3\%), amikacin (93.5\%), ceftazidime (90.3\%), azteronam (88.3\%) and cefepime (80.6\%). The nonESBL-producing group showed higher rates of susceptibility to most $\beta$-lactam antibiotics than did the ESBL-producing group; however, there was no significant difference in the rate of susceptibility to piperacillin/tazobactam between the two groups. Also, there were not significant differences in the rates of susceptibility to levofloxacin and trimethoprim for both groups; in contrast, the non-ESBL-producing group exhibited a higher rate of susceptibility to aminoglycosides (Table 1 ).

\section{Clinical characteristics and mechanisms of ESBL production in patients with $P$. mirabilis bacteremia}

The baseline characteristics of the patients are shown in Table 2. The median age was 74.5 years in the ESBL-producing group and 71 years in the non-ESBLproducing group. Patients in the ESBL-producing and non-ESBL-producing groups were predominantly male and female, respectively; however, this was not a significant difference. Urinary tract infection was the most frequent source of infection in the non-ESBLproducing group, while a significantly higher frequency of pneumonia, as a source of bacteremia, was seen in the ESBL-producing group $(42.9 \%$ vs. $6.3 \%$, $p=0.003)$.

Among the ESBL-producing strains, two produced TEM only, five produced CTX-M only, and six produced both TEM and CTX-M. One isolate showed a positive reaction in a double-disk potentiation test but did not express TEM-, CTX-M- or SHV-type enzymes.

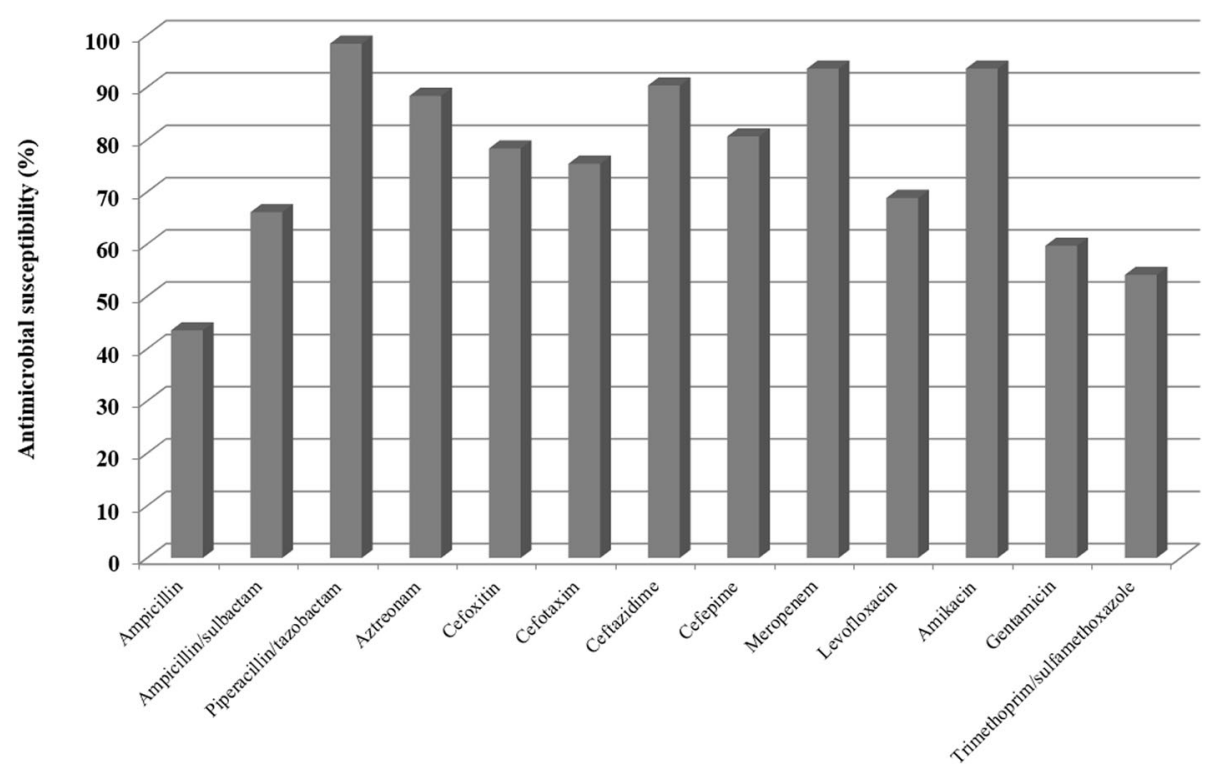

Fig. 1 In vitro antimicrobial susceptibility tests for Proteus mirabilis isolates causing bacteremia 
Table 1 In vitro antimicrobial susceptibility of ESBL producing and ESBL non-producing P. mirabilis isolates

\begin{tabular}{llll}
\hline Antimicrobial agent & No. (\%) of susceptible isolate & & $p$ value \\
\cline { 2 - 3 } & non-ESBL-producing group $N=48$ & ESBL producing groupN $=14$ \\
\hline Ampicillin & $26(54.2)$ & $1(7.1)$ & $4(28.6)$ \\
Ampicillin/sulbactam & $37(77.1)$ & $12(100)$ & 0.002 \\
Piperacillin/tazobactam & $47(97.9)$ & $8(61.5)$ & 0.003 \\
Aztreonam & $45(95.7)$ & $5(41.7)$ & $3(23.1)$ \\
Cefoxitin & $42(87.5)$ & $10(71.4)$ & 0.617 \\
Cefotaxim & $43(89.6)$ & $4(28.6)$ & 0.004 \\
Ceftazidime & $46(95.8)$ & $14(100)$ & 0.002 \\
Cefepime & $46(95.8)$ & $6(46.2)$ & 0.001 \\
Meropenem & $44(91.7)$ & $11(78.6)$ & 0.02 \\
Levofloxacin & $36(75.0)$ & $3(21.4)$ & 0.001 \\
Amikacin & $47(97.9)$ & $4(30.8)$ & 0.088 \\
Gentamicin & $34(70.8)$ & & 0.033 \\
Trimethoprim /sulfamethoxazole & $29(60.4)$ & & $0.057^{+}$ \\
\hline
\end{tabular}

ESBL, extended spectrum $\beta$-lactamases

\section{Factors associated with ESBL production by $P$. mirabilis}

A univariate analysis was conducted to investigate the factors associated with bacteremia caused by ESBLproducing $P$. mirabilis (Table 2). There were no significant differences in the rates of underlying comorbidities or most of the predisposing factors between the ESBLproducing and non-ESBL-producing groups. However, the rates of receiving intensive care unit (ICU) treatment and maintaining a percutaneous endoscopic gastrostomy (PEG) tube were significantly higher in the ESBLproducing group than in the non-ESBL-producing group (57.1\% vs. $22.9 \%, p=0.022$ for ICU, $28.6 \%$ vs. $2.1 \%$, $p=0.008$ for PEG tube). Initial clinical presentations and severity scores did not show meaningful differences between the two groups. However, in the multivariate analysis, no variable that was significant in the univariate analysis was independently associated with ESBL production (Table 3).

ESBL extended spectrum $\beta$-lactamases, PEG percutaneous endoscopic gastrostomy, ICU intensive care unit, $O R$ odd ratio, $C I$ confidence interval.

\section{Treatment outcomes of $P$. mirabilis bacteremia}

Treatment outcomes are also summarized in Table 2 . Inappropriate antibiotics were prescribed more frequently on the day of bacteremia diagnosis in the ESBLproducing group ( $42.9 \%$ vs. $18.8 \%, p=0.082)$, but the difference was not statistically significant. During the study period, 36 of 62 patients died, yielding an overall crude mortality rate of $58.6 \%$. Among these 36 patients, 11 died within 28 days of the $P$. mirabilis bacteremia episode, yielding a 28 -day mortality rate of $17.74 \%$. The ESBL-producing group showed a higher 28-day mortality rate than that of the non-ESBL-producing group
(50\% vs. $8.3 \%, p=0.001$ ); this was also confirmed by a survival curve analysis $(p=0.001)$ (Fig. 2).

\section{Factors associated with 28-day mortality in patients with $P$. mirabilis bacteremia}

Univariate and multivariate analyses of 51 survivors and 11 non-survivors were performed to identify the factors associated with 28-day mortality. As shown in Table 4, ESBL production by $P$. mirabilis, previous antibiotic use, and a higher baseline APACHE II score or SOFA score were significantly associated with 28-day mortality in patients with $P$. mirabilis bacteremia in the univariate analyses. In the multivariate analysis (Table 5), ESBL production (odds ratio [OR], 11.53; 95\% confidence interval $[\mathrm{CI}], 2.11-63.05, p=0.005)$ and a high SOFA score (OR 1.32, 95\% CI 1.03-1.70, $p=0.029$ ) were independently associated with 28-day mortality in patients with $P$. mirabilis bacteremia.

\section{Discussions}

Infections caused by Enterobacteriaceae expressing ESBLs are a healthcare concern worldwide; however, studies of ESBL production have focused on Klebsiella pneumoniae and Escherichia coli. [26-31] P. mirabilis is an important emerging pathogen, particularly in healthcare settings, due to its potential for horizontal transmission and drug resistance. [32] Reports on the antimicrobial resistance of $P$. mirabilis have increased in recent years. [32-35].

Four previous clinical studies on bacteremia caused by MDR $P$. mirabilis strains have addressed the risk factors for acquisition of antimicrobial resistance and the impact of MDR on mortality. [5, 8, 14, 15] Two of these studies focused on ESBL-producing strains $[8,15]$, while the other two evaluated MDR strains 
Table 2 Baseline characteristics and clinical outcomes of patients with P. mirabilis bacteremia

\begin{tabular}{|c|c|c|c|}
\hline Factors & $\begin{array}{l}\text { non-ESBL-producing group } \\
N=48\end{array}$ & $\begin{array}{l}\text { ESBL producing group } \\
N=14\end{array}$ & $p$ value \\
\hline Age, y, median(IQR) & $71.0(60.5-79)$ & $74.5(61.0-80.25)$ & 0.706 \\
\hline Age $\geq 65$ years, $n(\%)$ & $30(60.0)$ & $9(64.3)$ & 0.903 \\
\hline Male, n(\%) & $18(37.5)$ & $9(64.3)$ & 0.075 \\
\hline BMI, kg/m² median(IQR) & $22.37(20.51-25.54)$ & $21.21(18.80-23.46)$ & 0.247 \\
\hline \multicolumn{4}{|l|}{ Infection source } \\
\hline Urinary tract infection, n(\%) & $28(58.3)$ & $4(28.6)$ & 0.05 \\
\hline Pneumonia, n(\%) & $3(6.3)$ & $6(42.9)$ & 0.003 \\
\hline Skin and soft tissue infection, n(\%) & $1(2.1)$ & $1(7.1)$ & 0.403 \\
\hline Biliary infection, n(\%) & $9(18.8)$ & $0(0)$ & 0.105 \\
\hline Catheter related infection, n(\%) & $2(4.2)$ & $1(7.1)$ & 0.543 \\
\hline Others, n(\%) & $5(10.5)$ & $2(14.3)$ & 0.61 \\
\hline \multicolumn{4}{|l|}{ Comorbidities } \\
\hline HTN, n(\%) & $31(64.6)$ & $7(50)$ & 0.324 \\
\hline $\mathrm{DM}, \mathrm{n}(\%)$ & 19 (39.6) & $5(35.7)$ & 0.794 \\
\hline Cardiovascular disease, n(\%) & $4(8.3)$ & $4(28.6)$ & 0.069 \\
\hline Chronic kidney disease, n(\%) & $6(12.5)$ & $1(7.1)$ & 1.000 \\
\hline Chronic liver disease, n(\%) & $3(6.3)$ & $3(21.4)$ & 0.122 \\
\hline Solid tumor, n(\%) & $25(52.1)$ & $7(50)$ & 0.891 \\
\hline Hematologic malignancy, n(\%) & $2(4.2)$ & $0(0)$ & 1.000 \\
\hline Solid organ transplantation, n(\%) & $3(6.3)$ & $0(0)$ & 1.000 \\
\hline Charlson score, median(IQR) & $2.0(1.0-2.75)$ & $2.0(1.0-3.25)$ & 0.151 \\
\hline \multicolumn{4}{|l|}{ Predisposing factors } \\
\hline Neutropenia, n(\%) & $2(4.2)$ & $0(0)$ & 1.000 \\
\hline Chemotherapy, n(\%) & $5(10.4)$ & $3(21.4)$ & 0.365 \\
\hline Nursing home residence, n(\%) & $7(14.6)$ & $0(0)$ & 0.334 \\
\hline Hemodialysis, n(\%) & $7(14.6)$ & $4(28.6)$ & 0.249 \\
\hline ICU care, n(\%) & $11(22.9)$ & $8(57.1)$ & 0.022 \\
\hline Maintaining foley catheter, n(\%) & $11(22.9)$ & $6(42.9)$ & 0.141 \\
\hline Maintaining PEG tube, n(\%) & $1(2.1)$ & $4(28.6)$ & 0.008 \\
\hline Previous antibiotic use & $13(27.1)$ & $7(50)$ & 0.12 \\
\hline Cephalosporins, n(\%) & $6(12.5)$ & $2(14.3)$ & 1.000 \\
\hline Carbapenems, n(\%) & $2(4.2)$ & $2(4.2)$ & 0.217 \\
\hline Fluorquinolones, n(\%) & $2(4.2)$ & $1(7.1)$ & 0.543 \\
\hline BLBLIs, n(\%) & $4(8.4)$ & $1(7.1)$ & 1.000 \\
\hline \multicolumn{4}{|l|}{ Clinical presentation } \\
\hline Shock, n(\%) & $17(35.4)$ & $6(42.9)$ & 0.612 \\
\hline Acute kidney injury, n(\%) & $15(31.3)$ & $2(14.3)$ & 0.313 \\
\hline APACHE II score, median(IQR) & $12.0(8.0-16.75)$ & $13.5(10.75-19.25)$ & 0.115 \\
\hline SOFA score, median(IQR) & $3.0(1.0-5.75)$ & $4.0(1.0-6.5)$ & 0.85 \\
\hline \multicolumn{4}{|l|}{ Outcomes } \\
\hline Inappropriate antimicrobial therapy, n(\%) & $9(18.8)$ & $6(42.9)$ & 0.082 \\
\hline Overall mortality, n(\%) & $25(52.1)$ & $11(78.6)$ & 0.077 \\
\hline 28-day mortality, n(\%) & $4(8.3)$ & $7(50)$ & 0.001 \\
\hline
\end{tabular}

ESBL extended spectrum $\beta$-lactamases, IQR interquartile range, $B M I$ body mass index, HTN hypertension, DM diabetes mellitus, ICU intensive care unit, PEG percutaneous endoscopic gastrostomy, BLBLIs beta-lactam/beta-lactamase inhibitors, APACHE II score Acute Physiology and Chronic Health Evaluation II score, SOFA score the Sequential Organ Failure Assessment score 
Table 3 Multivariate analysis for associated factors of bacteremia caused by ESBL producing $P$. mirabilis

\begin{tabular}{llll}
\hline Factors & OR & $95 \% \mathrm{Cl}$ & $P$ value \\
\hline Age & 1.03 & $0.97-1.09$ & 0.294 \\
Female sex & 0.51 & $0.11-2.29$ & 0.375 \\
Having pneumonia as source of infection & 3.26 & $0.45-23.52$ & 0.241 \\
Maintaining PEG & 9.96 & $0.64-153.98$ & 0.101 \\
Previous ICU care & 3.15 & $0.69-14.29$ & 0.137 \\
\hline
\end{tabular}

defined as non-susceptible to at least one agent in three or more classes of antimicrobials. [5, 14] The risk factors for acquiring resistant $P$. mirabilis strains were receiving nursing home care $[5,8,14]$, previous antibiotic treatment $[5,14,15]$, hemodialysis [15], recurrent hospitalization [5, 14], urinary catheterization $[5,8]$ and having a peptic ulcer or peripheral vascular disease [14]. Only one of the four studies reported no differences in recurrence or mortality rates between MDR and non-MDR strains [15]; the others reported significantly higher mortality rates in patients with MDR P. mirabilis bacteremia. [5, 8, 14] In this study, no clinical factor was significantly associated with ESBL production and the 28-day mortality rate in the ESBL-producing group was significantly higher than that in the non-ESBL-producing group.

The prevalence of ESBLs in P. mirabilis varies among studies from $0.7 \%$ to $57 \%$ [15, 36-42]; however, many studies reported that the prevalence has increased over time. [36, 38-40] Previous studies from 2005 to 2011 in
Korea reported incidences of ESBL-producing $P$. mirabilis of $6.5-12.6 \%$, but no information regarding the change in incidence over time was provided. [16, 18, 43] In this study, the prevalence of ESBL production among P. mirabilis bacteremia isolates was $22.6 \%$ over an 8 -year period, which is higher than that reported by previous studies in Korea.

Most ESBLs are CTX-M-, TEM- and SHV-type $\beta$ lactamases. [31, 44] Recently, CTX-M-type $\beta$-lactamases have become the predominant type in many areas. [37, 38, $41,45,46]$ The distribution of ESBL type varies geographically; TEM-type enzymes are the most common ESBLs in some areas. [8, 40, 47, 48] According to one Korean study of ESBL-producing P. mirabilis isolates in 2005, most ESBL producers possessed the $b l a_{\mathrm{TEM}}$ gene,

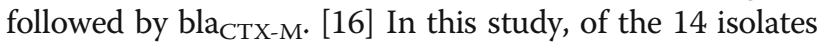
from the ESBL-producing group, 78.5\% (11/14) produced CTX-M, 50\% (7/14) produced TEM, and 49\% (6/14) produced both CTX-M and TEM; CTX-M type and TEM-type enzymes were also predominant in this study.

The $P$. mirabilis isolates showed a high rate of susceptibility to meropenem. Only 4 of 62 strains were not susceptible to meropenem, all of which were in the nonESBL-producing group. Among them, one strain isolated in 2012 showed intermediate susceptibility to meropenem minimum inhibitory concentration (MIC) $2 \mathrm{mg} / \mathrm{L}$ but was susceptible to piperacillin/tazobactam and other third- and fourth-generation cephalosporins. The other three strains (two strains with meropenem disk zone diameters of $21 \mathrm{~mm}$ and one strain with meropenem MIC

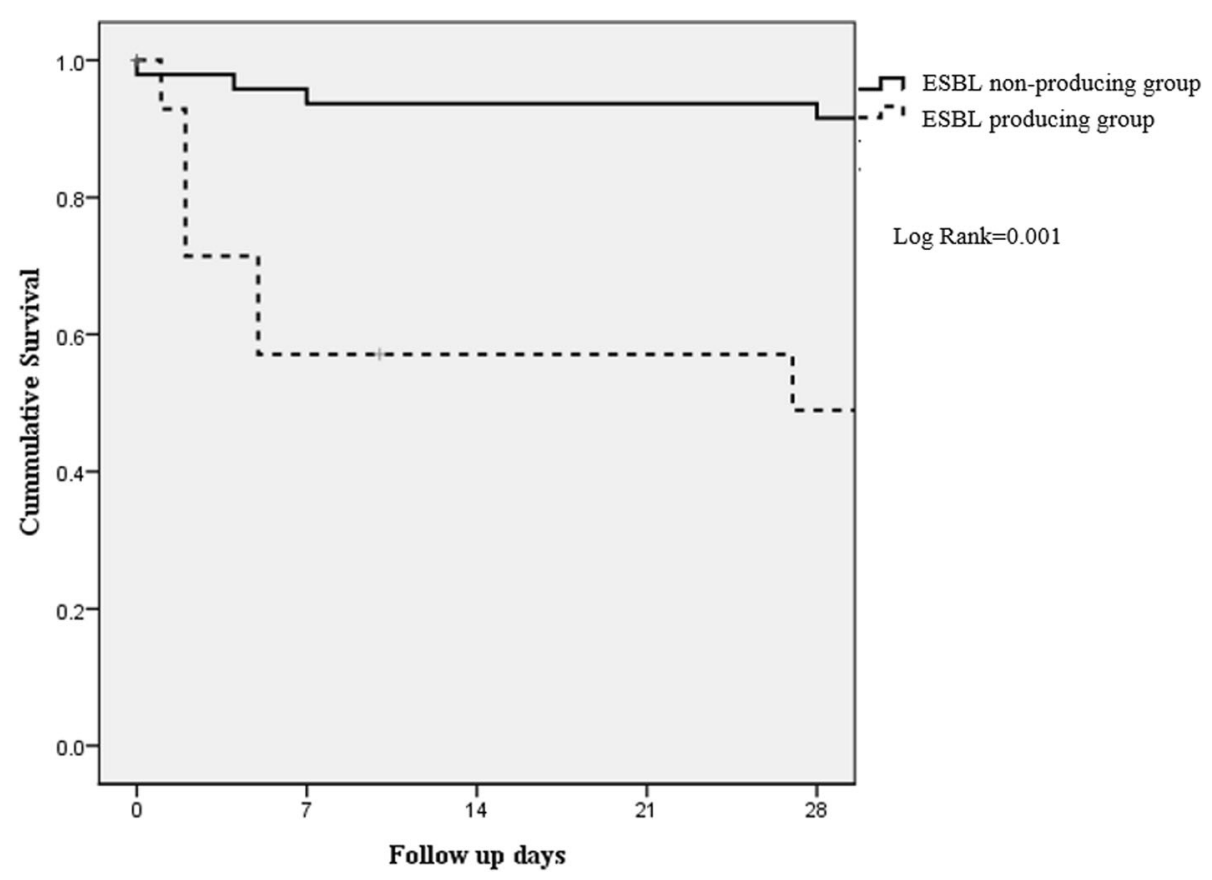

Fig. 2 Kaplan-Meier survival estimates among patients with P. mirabilis bacteremia. ESBL, extended spectrum $\beta$-lactam 
Table 4 Factors associated with 28-day mortality in patients with $P$. mirabilis bacteremia

\begin{tabular}{|c|c|c|c|}
\hline Factors & Survivors $N=51(\%)$ & Nonsurvivors N = 11 (\%) & $p$ value \\
\hline Age, y, median(IQR) & $71.0(63.0-79.0)$ & $73.0(54.0-84.0)$ & 0.797 \\
\hline Age $\geq 65$ years, $n(\%)$ & $32(62.7)$ & $7(63.6)$ & 1.000 \\
\hline Sex, male, n(\%) & $21(41.2)$ & $6(54.6)$ & 0.51 \\
\hline BMI, kg/m², median(IQR) & $22.35(10.07-25.52)$ & $21.78(19.15-23.29)$ & 0.366 \\
\hline ESBL producing pathogen, yes, $n(\%)$ & $7(13.7)$ & $7(63.6)$ & 0.001 \\
\hline \multicolumn{4}{|l|}{ Infection source } \\
\hline Urinary tract infection, n(\%) & $28(54.9)$ & $4(36.4)$ & 0.264 \\
\hline Pneumonia, n(\%) & $6(11.8)$ & $3(27.3)$ & 0.191 \\
\hline Skin and soft tissue infection, n(\%) & $2(3.9)$ & $0(0)$ & 1.000 \\
\hline Biliary infection, n(\%) & $8(15.7)$ & $1(9.1)$ & 1.000 \\
\hline Catheter related infection, n(\%) & $2(3.9)$ & $1(9.1)$ & 0.449 \\
\hline \multicolumn{4}{|l|}{ Comorbities } \\
\hline HTN, n(\%) & $33(64.7)$ & $5(45.5)$ & 0.311 \\
\hline $\mathrm{DM}, \mathrm{n}(\%)$ & $21(41.2)$ & $3(27.3)$ & 0.505 \\
\hline Cardiovascular disease, n(\%) & $5(9.8)$ & $3(27.3)$ & 0.142 \\
\hline Chronic kidney disease, n(\%) & $7(13.7)$ & $0(0)$ & 0.334 \\
\hline Chronic liver disease, n(\%) & $3(5.9)$ & $3(27.3)$ & 0.063 \\
\hline Rheumatologic disease, n(\%) & $2(3.9)$ & $0(0)$ & 1.000 \\
\hline Solid tumor, n(\%) & $26(51.0)$ & $6(54.5)$ & 0.83 \\
\hline Hematologic malignancy, n(\%) & $1(2.0)$ & $1(9.1)$ & 0.326 \\
\hline Solid organ transplantation, n(\%) & $2(3.9)$ & $1(9.1)$ & 0.449 \\
\hline Charlson score, median(IQR) & $2.0(1.0-2.0)$ & $2.0(1.0-4.0)$ & 0.239 \\
\hline \multicolumn{4}{|l|}{ Predisposing factors } \\
\hline Neutropenia, n(\%) & $2(4.0)$ & $0(0)$ & 1.000 \\
\hline Chemotherapy, n(\%) & $7(13.7)$ & $1(9.1)$ & 1.000 \\
\hline Nursing home residence, n(\%) & $7(13.7)$ & $0(0)$ & 0.334 \\
\hline Hemodialysis, n(\%) & $8(15.7)$ & $3(27.3)$ & 0.394 \\
\hline Maintaining foley catheter, n(\%) & $11(21.6)$ & $6(54.5)$ & 0.056 \\
\hline Maintaining PEG tube, n(\%) & $4(7.8)$ & $1(9.1)$ & 1.000 \\
\hline ICU care, n(\%) & $13(25.5)$ & $6(54.5)$ & 0.077 \\
\hline Previous antibiotic use, n(\%) & $13(25.5)$ & $7(63.6)$ & 0.029 \\
\hline Cephalosporins, n(\%) & $6(11.8)$ & $2(18.2)$ & 0.623 \\
\hline Carbapenems, n(\%) & $3(5.9)$ & $1(9.1)$ & 0.552 \\
\hline Fluorquinolones, n(\%) & $1(2.0)$ & $2(18.2)$ & 0.079 \\
\hline BLBLI, n(\%) & $3(5.9)$ & $2(18.2)$ & 0.212 \\
\hline \multicolumn{4}{|l|}{ Clinical presentation } \\
\hline Shock, n(\%) & $19(37.3)$ & $4(36.4)$ & 1.000 \\
\hline Acute kidney injury, n(\%) & $14(27.5)$ & $3(27.3)$ & 1.000 \\
\hline APACHE II score, median(IQR) & $11.0(8.0-16.0)$ & $17.0(11.0-19.0)$ & 0.027 \\
\hline SOFA score, median(IQR) & $2.0(1.0-5.0)$ & $5.0(4.0-8.0)$ & 0.033 \\
\hline Inappropriate antimicrobial therapy, n(\%) & 10 (19.6) & $5(45.5)$ & 0.115 \\
\hline
\end{tabular}

IQR interquartile range, $B M I$ body mass index, ESBL extended spectrum $\beta$-lactamases, $H T N$ hypertension, DM diabetes mellitus, PEG percutaneous endoscopic gastrostomy, ICU intensive care unit, BLBLIs beta-lactam/beta-lactamase inhibitors, APACHE II score Acute Physiology and Chronic Health Evaluation II score, SOFA score the Sequential Organ Failure Assessment score 
Table 5 Multivariate analysis of risk factors for 28-day mortality

\begin{tabular}{llll}
\hline Factors & OR & $95 \% \mathrm{Cl}$ & $p$ value \\
\hline ESBL producing & 11.53 & $2.11-63.05$ & 0.005 \\
Previous antibiotics use & 5.09 & $0.94-27.54$ & 0.059 \\
SOFA score & 1.32 & $1.03-1.69$ & 0.029
\end{tabular}

ESBL extended spectrum $\beta$-lactamases, SOFA score the Sequential Organ Failure Assessment score, $O R$ odd ratio, $\mathrm{Cl}$ confidence interval

of $4 \mathrm{mg} / \mathrm{L}$ ) were isolated between 2008 and 2009 and were deemed susceptible prior to the retrospective adjustment according to the modified CLSI guidelines. [19] The carbapenem-resistant isolate (one strain with meropenem MIC of $4 \mathrm{mg} / \mathrm{L}$ ) showed decreased susceptibility to ampicillin, ampicillin/sulbactam, aminoglycosides, quinolones and trimethoprim/sulfamethoxazole but was susceptible to piperacillin/tazobactam and other thirdand fourth-generation cephalosporins.

Almost all $P$. mirabilis isolates were susceptible to piperacillin/tazobactam, irrespective of ESBL production. Piperacillin/tazobactam is less effective than meropenem against bacteremia caused by ESBL-producing Enterobacteriaceae [49-51]. Therefore, piperacillin/tazobactam can be used empirically prior to determination of the susceptibility profile of potential or predicted ESBLproducing $P$. mirabilis isolates.

In this study, the ESBL-producing group showed a significantly higher 28-day mortality rate than that of the non-ESBL-producing group. The prognosis of bacteremia caused by Enterobacteriaceae is associated with several factors, such as the clinical severity at the time of antimicrobial treatment, underlying diseases and the appropriateness of antibiotics. [52] The higher rate of inappropriate initial antimicrobial treatment in patients with ESBL-producing $P$. mirabilis could explain the increased mortality rate. $[5,26,53$, 54] However, in this study, clinical severity, underlying diseases and the rate of receiving inappropriate initial antibiotics did not differ between the ESBLproducing and non-ESBL-producing groups. Although the analyses were not limited to $P$. mirabilis infection, worse clinical outcomes from infections caused by ESBL-producing pathogens susceptible to the antibiotics used have been reported. $[52,55,56] \mathrm{Kim}$ et al. reported that the favorable treatment response rate was significantly lower in the ESBL group than in the non-ESBL group among 68 patients with bacteremia caused by $E$. coli or $K$. pneumoniae treated with extended-spectrum cephalosporins, to which the infecting organisms were susceptible in vitro. [52] The mean interval from the time of bacteremia to the administration of presumptively appropriate antimicrobial agents was not different between the two groups. [52] Although the research did not make use of the revised cephalosporin breakpoints, the authors concluded that ESBL production itself resulted in a worse prognosis of bacteremia. [52] Use of certain extended-spectrum cephalosporins for infections caused by ESBL-producing pathogens can result in treatment failure. In particular, a favorable response to treatment with a third-generation cephalosporin other than ceftazidime has been reported in some cases of infection with ESBL-producing strains. [27] Moreover, the molecular type of ESBL can also affect the treatment outcomes. [27, 57, 58] Finally, an inoculum effect, i.e., a significant increase in the MIC with an increasing number of organisms, has been suggested to explain the worse prognosis associated with infections caused by ESBL-producing pathogens treated with antibiotics other than carbapenems [55].

Infections with ESBL-producing strains can be treated early with appropriate antibiotics before the availability of antimicrobial susceptibility profiles in the presence of clinical risk factors predictive of infection with ESBLproducing strains. However, no clinical factor was significantly associated with ESBL production in this study. Therefore, rapid detection of ESBL expression is essential for early appropriate treatment. Microbiologic identification using matrix-assisted laser desorption/ ionization time-of-flight mass spectrometry and rapid susceptibility testing facilitates faster detection of pathogens and prompt prescription of appropriate antibiotics, which may lead to improved clinical outcomes [59-61].

This study was subject to several limitations. First, the type of CTX-M and TEM $\beta$-lactamases was not identified, and ESBL-producing strains were not subjected to pulsed-field gel electrophoresis assay. Therefore, the ESBL types and role of horizontal spread of their encoding genes could not be assessed. Next, stored $P$. mirabilis isolate samples were available for only 64 of 85 patients infected with $P$. mirabilis bacteremia during the study period. Therefore, we were unable to evaluate the incidence of ESBL-producing isolates over time. Other limitations include the retrospective nature and small sample size of the study. Nevertheless, our findings suggest that ESBL production exerts a negative influence on the mortality rate of patients with $P$. mirabilis bacteremia.

\section{Conclusions}

In conclusion, our results suggest that ESBL production is significantly associated with the 28-day mortality rate, in that patients with bacteremia caused by ESBLproducing $P$. mirabilis have higher 28-day mortality rates. Because no clinical factor was found to be predictive of ESBL production by $P$. mirabilis bacteremia isolates, early detection of ESBL expression and prompt appropriate antimicrobial therapy are essential for improving the prognosis. 


\section{Abbreviations}

APACHE: acute physiological and chronic health evaluation; $\mathrm{Cl}$ : confidence interval; CLSI: Clinical and Laboratory Standards Institute; ESBL: extendedspectrum $\beta$-lactamase; ICU: intensive care unit; IQR: interquartile range; IRB: Institutional Review Board; MDR: multidrug-resistant; MIC: minimum inhibitory concentration; OR: odds ratio; PEG: percutaneous endoscopic gastrostomy; SD: standard deviation; SOFA: sequential organ failure

\section{Acknowledgements}

Not applicable.

\section{Funding}

This research received no specific grant from any funding agency in the public, commercial, or not-for-profit sectors.

\section{Availability of data and materials}

All data generated or analyzed during this study are included in this published article and supplementary information files.

\section{Authors' contributions}

All authors (JYA, HWA, YJ, MYA, DHO, YCK, EJK, JES, IYJ, MHK, WJ, NSK, SJJ, JYC, DY, YGS, JMK) have read and approved the final manuscript. All authors have made contributions to conception of this study and have been involved in revising the manuscript and have given final approval of the version to be published. JYA and NSK have been involved in drafting the manuscript, HWA, YJ, MYA, IYJ, JYC, JMK have made contributions to analysis and interpretation of data and DHO, YCK, EJK, JES, MHK, WJ, SJJ, DY, YGS, JMK have made contributions to acquisition of data.

\section{Competing interests}

All authors reported no disclosures and competing interests.

\section{Consent for publication}

Not applicable.

\section{Ethics approval and consent to participate}

The study was approved by the Institutional Review Board (IRB) of Yonse University Health System Clinical Trial Center. Since the study was retrospective, and the data of the subjects were anonymized, the IRB waived the requirement for written informed consent from the patients.

\section{Publisher's Note}

Springer Nature remains neutral with regard to jurisdictional claims in published maps and institutional affiliations.

\section{Author details}

'Department of Internal Medicine, Seoul Medical Center, Seoul, South Korea. ${ }^{2}$ Department of Internal Medicine, Yonsei University College of Medicine, Seoul, South Korea. ${ }^{3}$ AIDS Research Institute, Yonsei University College of Medicine, Seoul, South Korea. ${ }^{4}$ Department of Internal Medicine, Inje University College of Medicine, Ilsan Paik Hospital, Goyang, South Korea. ${ }^{5}$ Department of Laboratory Medicine, Yonsei University College of Medicine, Seoul, South Korea. ${ }^{6}$ Department of Internal Medicine and AIDS Research Institute, Yonsei University College of Medicine, 50-1 Yonsei-ro,

Seodaemun-gu, Seoul 120-752, South Korea.

Received: 1 January 2017 Accepted: 28 April 2017

Published online: 03 May 2017

\section{References}

1. Diekema DJ, Pfaller MA, Jones RN, Doern GV, Kugler KC, Beach ML, Sader HS. Trends in antimicrobial susceptibility of bacterial pathogens isolated from patients with bloodstream infections in the USA, Canada and Latin America. SENTRY participants group. Int J Antimicrob Agents. 2000;13(4):257-71.

2. Sader HS, Jones RN, Andrade-Baiocchi S, Biedenbach DJ, Group SP. Four-year evaluation of frequency of occurrence and antimicrobial susceptibility patterns of bacteria from bloodstream infections in Latin American medical centers. Diagn Microbiol Infect Dis. 2002;44(3):273-80.

3. Luzzaro F, Ortisi G, Larosa M, Drago M, Brigante G, Gesu G. Prevalence and epidemiology of microbial pathogens causing bloodstream infections: results of the OASIS multicenter study. Diagn Microbiol Infect Dis. 2011:69(4):363-9.

4. Watanakunakorn C, Perni SC. Proteus Mirabilis bacteremia: a review of 176 cases during 1980-1992. Scand J Infect Dis. 1994;26(4):361-7.

5. Tumbarello M, Trecarichi EM, Fiori B, Losito AR, D'Inzeo T, Campana L, Ruggeri A, Di Meco E, Liberto E, Fadda G, et al. Multidrug-resistant Proteus Mirabilis bloodstream infections: risk factors and outcomes. Antimicrob Agents Chemother. 2012;56(6):3224-31.

6. Jacobsen SM, Stickler DJ, Mobley HL, Shirtliff ME. Complicated catheterassociated urinary tract infections due to Escherichia coli and Proteus Mirabilis. Clin Microbiol Rev. 2008;21(1):26-59.

7. Karlowsky JA, Jones ME, Thornsberry C, Friedland IR, Sahm DF. Trends in antimicrobial susceptibilities among Enterobacteriaceae isolated from hospitalized patients in the United States from 1998 to 2001. Antimicrob Agents Chemother. 2003;47(5):1672-80

8. Endimiani A, Luzzaro F, Brigante G, Perilli M, Lombardi G, Amicosante G, Rossolini GM, Toniolo A. Proteus Mirabilis bloodstream infections: risk factors and treatment outcome related to the expression of extended-spectrum beta-lactamases. Antimicrob Agents Chemother. 2005:49(7):2598-605.

9. Cohen-Nahum K, Saidel-Odes L, Riesenberg K, Schlaeffer F, Borer A. Urinary tract infections caused by multi-drug resistant Proteus Mirabilis: risk factors and clinical outcomes. Infection. 2010;38(1):41-6.

10. D'Andrea MM, Literacka E, Zioga A, Giani T, Baraniak A, Fiett J, Sadowy E, Tassios PT, Rossolini GM, Gniadkowski M, et al. Evolution and spread of a multidrugresistant Proteus Mirabilis clone with chromosomal AmpC-type cephalosporinases in Europe. Antimicrob Agents Chemother. 2011;55(6):2735-42.

11. Luzzaro F, Brigante G, D'Andrea MM, Pini B, Giani T, Mantengoli E, Rossolini GM, Toniolo A. Spread of multidrug-resistant Proteus Mirabilis isolates producing an AmpC-type beta-lactamase: epidemiology and clinical management. Int J Antimicrob Agents. 2009;33(4):328-33.

12. Pagani L, Migliavacca R, Pallecchi L, Matti C, Giacobone E, Amicosante G, Romero E, Rossolini GM. Emerging extended-spectrum beta-lactamases in Proteus Mirabilis. J Clin Microbiol. 2002;40(4):1549-52.

13. Tsakris A, Ikonomidis A, Poulou A, Spanakis N, Pournaras S, Markou F. Transmission in the community of clonal Proteus Mirabilis carrying VIM-1 metallo-beta-lactamase. J Antimicrob Chemother. 2007:60(1):136-9.

14. Korytny A, Riesenberg K, Saidel-Odes L, Schlaeffer F, Borer A. Bloodstream infections caused by multi-drug resistant Proteus Mirabilis: epidemiology, risk factors and impact of multi-drug resistance. Infect Dis (Lond). 2016:48(6):428-31.

15. Kurihara $Y$, Hitomi S, Oishi T, Kondo T, Ebihara T, Funayama Y, Kawakami Y. Characteristics of bacteremia caused by extended-spectrum beta-lactamaseproducing Proteus Mirabilis. J Infect Chemother. 2013;19(5):799-805.

16. Park YJ, Lee S, Kim YR, Oh EJ, Woo GJ, Lee K. Occurrence of extended-spectrum (beta)-lactamases and plasmid-mediated AmpC (beta)-lactamases among Korean isolates of Proteus Mirabilis. J Antimicrob Chemother. 2006:57(1):156-8.

17. Park SD, Uh Y, Lee G, Lim K, Kim JB, Jeong SH. Prevalence and resistance patterns of extended-spectrum and AmpC beta-lactamase in Escherichia coli, Klebsiella pneumoniae, Proteus Mirabilis, and salmonella serovar Stanley in a Korean tertiary hospital. APMIS. 2010;118(10):801-8.

18. Song W, Kim J, Bae IK, Jeong SH, Seo YH, Shin JH, Jang SJ, Uh Y, Shin JH, Lee MK, et al. Chromosome-encoded AmpC and CTX-M extended-spectrum beta-lactamases in clinical isolates of Proteus Mirabilis from Korea. Antimicrob Agents Chemother. 2011:55(4):1414-9.

19. CLSI. Performance Standards for antimicrobial susceptibility testing; twentyfourth informational supplement. CLSI document M100-S24. Clinical and Laboratory Standards Institute: Wayne; 2014.

20. CLSI. Performance Standards for antimicrobial susceptibility testing; twentyfourth informational supplement. CLSI document M100-S21. Clinical and Laboratory Standards Institute: Wayne; 2011.

21. Lee $\mathrm{K}$, Yong D, Yum JH, Kim HH, Chong Y. Diversity of TEM-52 extendedspectrum beta-lactamase-producing non-typhoidal salmonella isolates in Korea J Antimicrob Chemother. 2003:52(3):493-6.

22. Chong $Y$, Lee $K$, Okamoto $R$, Inoue $M$. Characteristics of extendedspectrum $\beta$-lactam hydrolyzing activity of Klebsiella pneumoniae and Escherichia coli strains isolated from clinical specimens. Korean J Infect Dis. 1997:29(6):477-86.

23. Park Y, Kang HK, Bae IK, Kim J, Kim JS, Uh Y, Jeong SH, Lee K. Prevalence of the extended-spectrum beta-lactamase and anr genes in clinical isolates of Escherichia coli. Korean J Lab Med. 2009;29(3):218-23. 
24. Kang Cl, Kim SH, Park WB, Lee KD, Kim HB, Oh MD, Kim EC, Choe KW. Bloodstream infections caused by Enterobacter species: predictors of 30-day mortality rate and impact of broad-spectrum cephalosporin resistance on outcome. Clin Infect Dis. 2004;39(6):812-8.

25. International Classification of Disease. World Health Organization. 2014

26. Schwaber MJ, Carmeli Y. Mortality and delay in effective therapy associated with extended-spectrum beta-lactamase production in Enterobacteriaceae bacteraemia: a systematic review and meta-analysis. J Antimicrob Chemother. 2007;60(5):913-20.

27. Wong-Beringer A, Hindler J, Loeloff M, Queenan AM, Lee N, Pegues DA, Quinn JP, Bush K. Molecular correlation for the treatment outcomes in bloodstream infections caused by Escherichia coli and Klebsiella pneumoniae with reduced susceptibility to ceftazidime. Clin Infect. 2002;34(2):135-46.

28. Kim BN, Woo JH, Kim MN, Ryu J, Kim YS. Clinical implications of extendedspectrum beta-lactamase-producing Klebsiella pneumoniae bacteraemia. J Hosp Infect. 2002;52(2):99-106.

29. Du B, Long Y, Liu H, Chen D, Liu D, Xu Y, Xie X. Extended-spectrum beta-lactamase-producing Escherichia coli and Klebsiella pneumoniae bloodstream infection: risk factors and clinical outcome. Intensive Care Med. 2002;28(12):1718-23.

30. Menashe G, Borer A, Yagupsky P, Peled N, Gilad J, Fraser D, Riesenberg K, Schlaeffer $F$. Clinical significance and impact on mortality of extendedspectrum beta lactamase-producing Enterobacteriaceae isolates in nosocomial bacteremia. Scand J Infect Dis. 2001;33(3):188-93.

31. Bradford PA. Extended-spectrum beta-lactamases in the 21 st century: characterization, epidemiology, and detection of this important resistance threat. Clin Microbiol Rev. 2001;14(4):933-51. table of contents

32. Nagano N, Shibata N, Saitou Y, Nagano Y, Arakawa Y. Nosocomial outbreak of infections by Proteus Mirabilis that produces extended-spectrum CTX-M2 type beta-lactamase. J Clin Microbiol. 2003;41(12):5530-6.

33. Kim JY, Park YJ, Kim SI, Kang MW, Lee SO, Lee KY. Nosocomial outbreak by Proteus Mirabilis producing extended-spectrum beta-lactamase VEB-1 in a Korean university hospital. J Antimicrob Chemother. 2004;54(6):1144-7.

34. Nakano R, Nakano A, Abe M, Inoue M, Okamoto R. Regional outbreak of CTX-M-2 beta-lactamase-producing Proteus Mirabilis in Japan. J Med Microbiol. 2012;61(Pt 12):1727-35.

35. de Champs C, Bonnet R, Sirot D, Chanal C, Sirot J. Clinical relevance of Proteus Mirabilis in hospital patients: a two year survey. J Antimicrob Chemother. 2000;45(4):537-9.

36. Nakamura T, Komatsu M, Yamasaki K, Fukuda S, Miyamoto Y, Higuchi T, Ono T, Nishio H, Sueyoshi N, Kida K, et al. Epidemiology of Escherichia coli, Klebsiella species, and Proteus Mirabilis strains producing extendedspectrum beta-lactamases from clinical samples in the Kinki region of Japan. Am J Clin Pathol. 2012;137(4):620-6.

37. Wang JT, Chen PC, Chang SC, Shiau YR, Wang HY, Lai JF, Huang IW, Tan MC, Lauderdale TL, Hospitals T. Antimicrobial susceptibilities of Proteus Mirabilis: a longitudinal nationwide study from the Taiwan surveillance of antimicrobial resistance (TSAR) program. BMC Infect Dis. 2014;14:486.

38. Ho PL, Ho AY, Chow KH, Wong RC, Duan RS, Ho WL, Mak GC, Tsang KW, Yam WC, Yuen KY. Occurrence and molecular analysis of extendedspectrum \{beta\}-lactamase-producing Proteus Mirabilis in Hong Kong, 1999-2002. J Antimicrob Chemother. 2005:55(6):840-5.

39. Wu JJ, Chen HM, Ko WC, Wu HM, Tsai SH, Yan JJ. Prevalence of extendedspectrum beta-lactamases in Proteus Mirabilis in a Taiwanese university hospital, 1999 to 2005: identification of a novel CTX-M enzyme (CTX-M-66) Diagn Microbiol Infect Dis. 2008;60(2):169-75.

40. Tonkic M, Mohar B, Sisko-Kraljevic K, Mesko-Meglic K, Goic-Barisic I, Novak A, Kovacic A, Punda-Polic V. High prevalence and molecular characterization of extended-spectrum beta-lactamase-producing Proteus Mirabilis strains in southern Croatia. J Med Microbiol. 2010;59(Pt 10):1185-90.

41. Kanayama A, Kobayashi I, Shibuya K. Distribution and antimicrobial susceptibility profile of extended-spectrum beta-lactamase-producing Proteus Mirabilis strains recently isolated in Japan. Int J Antimicrob Agents. 2015;45(2):113-8.

42. Rudresh SM, Nagarathnamma T. Extended spectrum beta-lactamase producing Enterobacteriaceae \& antibiotic co-resistance. Indian J Med Res. 2011;133:116-8.

43. Uh Y, Hwang GY, Kwon O, Yoon KJ, Kim HY. Isolation frequency of extended spectrum b-lactamase producing Escherichia coli, Klebsiella species, and Proteus Mirabilis. Korean J Clin Microbiol. 2007;10(2):119-22.
44. Livermore DM, Woodford $\mathrm{N}$. The beta-lactamase threat in Enterobacteriaceae, pseudomonas and Acinetobacter. Trends Microbiol. 2006;14(9):413-20

45. Bonnet R. Growing group of extended-spectrum beta-lactamases: the CTX-M enzymes. Antimicrob Agents Chemother. 2004;48(1):1-14.

46. Livermore DM, Canton R, Gniadkowski M, Nordmann P, Rossolini GM, Arlet G, Ayala J, Coque TM, Kern-Zdanowicz I, Luzzaro F, et al. CTX-M: changing the face of ESBLs in Europe. J Antimicrob Chemother. 2007;59(2):165-74.

47. Biendo M, Thomas D, Laurans G, Hamdad-Daoudi F, Canarelli B, Rousseau F, Castelain S, Eb F. Molecular diversity of Proteus Mirabilis isolates producing extended-spectrum beta-lactamases in a French university hospital. Clin Microbiol Infect. 2005;11(5):395-401.

48. Spanu T, Luzzaro F, Perilli M, Amicosante G, Toniolo A, Fadda G, Italian ESG. Occurrence of extended-spectrum beta-lactamases in members of the family Enterobacteriaceae in Italy: implications for resistance to beta-lactams and other antimicrobial drugs. Antimicrob Agents Chemother. 2002;46(1):196-202.

49. Tsai HY, Chen YH, Tang HJ, Huang CC, Liao CH, Chu FY, Chuang YC, Sheng WH, Ko WC, Hsueh PR. Carbapenems and piperacillin/tazobactam for the treatment of bacteremia caused by extended-spectrum beta-lactamaseproducing Proteus Mirabilis. Diagn Microbiol Infect Dis. 2014;80(3):222-6.

50. Tamma PD, Han JH, Rock C, Harris AD, Lautenbach E, Hsu AJ, Avdic E, Cosgrove SE, Antibacterial Resistance Leadership G. Carbapenem therapy is associated with improved survival compared with piperacillin-tazobactam for patients with extended-spectrum beta-lactamase bacteremia. Clin Infect Dis. 2015;60(9):1319-25.

51. Ofer-Friedman H, Shefler C, Sharma S, Tirosh A, Tal-Jasper R, Kandipalli D, Sharma S, Bathina P, Kaplansky T, Maskit M, et al. Carbapenems versus Piperacillin-Tazobactam for bloodstream infections of Nonurinary source caused by extended-Spectrum Beta-Lactamase-producing Enterobacteriaceae. Infect Control Hosp Epidemiol. 2015;36(8):981-5.

52. Kim YK, Pai H, Lee HJ, Park SE, Choi EH, Kim J, Kim JH, Kim EC. Bloodstream infections by extended-spectrum beta-lactamase-producing Escherichia coli and Klebsiella pneumoniae in children: epidemiology and clinical outcome. Antimicrob Agents Chemother. 2002;46(5):1481-91.

53. Giske CG, Monnet DL, Cars O, Carmeli Y, ReAct-Action on Antibiotic R. Clinical and economic impact of common multidrug-resistant gramnegative bacilli. Antimicrob Agents Chemother. 2008;52(3):813-21.

54. Maslikowska JA, Walker SA, Elligsen M, Mittmann N, Palmay L, Daneman N, Simor A. Impact of infection with extended-spectrum beta-lactamaseproducing Escherichia coli or Klebsiella species on outcome and hospitalization costs. J Hosp Infect. 2016;92(1):33-41.

55. Ramphal R, Ambrose PG. Extended-spectrum beta-lactamases and clinical outcomes: current data. Clin Infect Dis. 2006;42(Suppl 4):S164-72.

56. Henshke-Bar-Meir R, Yinnon AM, Rudensky B, Attias D, Schlesinger $Y$, Raveh D. Assessment of the clinical significance of production of extended-spectrum beta-lactamases (ESBL) by Enterobacteriaceae. Infection. 2006;34(2):66-74.

57. Rasheed JK, Anderson GJ, Queenan AM, Biddle JW, Oliver A, Jacoby GA, Bush K, Tenover FC. TEM-71, a novel plasmid-encoded, extended-spectrum beta-lactamase produced by a clinical isolate of Klebsiella pneumoniae. Antimicrob Agents Chemother. 2002;46(6):2000-3.

58. Ma L, Ishii Y, Chang FY, Yamaguchi K, Ho M, Siu LK. CTX-M-14, a plasmidmediated CTX-M type extended-spectrum beta-lactamase isolated from Escherichia coli. Antimicrob Agents Chemother. 2002;46(6):1985-8.

59. Dixon P, Davies P, Hollingworth W, Stoddart M, MacGowan A. A systematic review of matrix-assisted laser desorption/ionisation time-of-flight mass spectrometry compared to routine microbiological methods for the time taken to identify microbial organisms from positive blood cultures. Eur J Clin Microbiol Infect Dis. 2015;34(5):863-76.

60. Verroken A, Defourny L, le Polain de Waroux O, Belkhir L, Laterre PF, Delmee M, Glupczynski Y. Clinical impact of MALDI-TOF MS identification and rapid susceptibility testing on adequate antimicrobial treatment in sepsis with positive blood cultures. PLoS One. 2016;11(5):e0156299.

61. Lin WH, Hwang JC, Tseng CC, Chang YT, Wu AB, Yan JJ, Wu JJ, Wang MC. Matrix-assisted laser desorption ionization-time of flight mass spectrometry accelerates pathogen identification and may confer benefit in the outcome of peritoneal dialysis-related peritonitis. J Clin Microbiol. 2016;54(5):1381-3. 